INTERNACIONAL

\title{
Juzgar con perspectiva de género: Fundamentos y análisis de sentencias
}

\author{
Judge with gender perspective: Foundations and analysis of judgments
}

\author{
Pilar Maturana \\ Universidad Católica del Norte, Chile
}

\begin{abstract}
RESUMEN Las promesas de igualdad, libertad y solidaridad se mantienen incumplidas para la mitad de la humanidad, para las excluidas, las de los márgenes: las mujeres. ¿Cómo cambiamos esa realidad? Si bien el derecho ha dado respuesta a los derechos de las mujeres, al concretizar aquéllos consagrados de manera universal, su incorporación no ha sido suficiente para cumplir las promesas de la modernidad. Por ello, este artículo se detiene en la incorporación de la categoría género en las sentencias en perspectiva de género, como una técnica con la que hacer frente a las desigualdades entre hombres y mujeres. Desde ese punto de análisis, se referirá luego a las implicancias que ha tenido la incorporación de la perspectiva de género en sentencias de la Corte Interamericana de Derechos Humanos, y en un fallo de la Corte Suprema chilena que incorpora y desarrolla esta categoría.
\end{abstract}

PALABRAS CLAVE Género, perspectiva de género, sentencias judiciales, jueces y juezas.

ABSTRACT The promises of equality, freedom and solidarity remain unfulfilled for half of humanity, for the excluded, those on the margins: women. How do we change that reality? Although the Law has responded to the rights of women, concretizing those universally consecrated, its incorporation has not been enough to fulfill the promises of modernity. That's why this article analyzes the incorporation of the gender category in judgments, with gender mainstreaming as a technique with which to deal with the inequalities between men and women and the discrimination of the last. From that point of analysis, it will go on to refer to the implications that the incorporation of the gender perspective has had in the judgments by the Inter-American Court of Human Rights, grouped in three contributions that we want to highlight and in a decision of the Chilean Supreme Court that incorporates and develops this category.

KEYWORDS: Gender, gender perspective, judgments, judges and judges. 


\section{Cuando el género suena, consecuencias trae: La incorporación de la perspectiva de género en las sentencias}

Superada la decimonónica idea de que la labor de los jueces y juezas es de ser puramente la boca muda que pronuncia las palabras de la ley ${ }^{1}$-sin que ello quiera decir la no vinculación a ese derecho- ${ }^{2}$ como un pilar básico en los Estados constitucionales, en cuanto garantes de los derechos fundamentales ${ }^{3}$ que, como conquista histórica, constituyen un elemento esencial del sistema de legitimidad en el que se apoya el Estado de derecho y el ejercicio del poder, reconociendo además que las sentencias «tienen un poder individual y colectivo que impacta en la vida de las personas y conforman la identidad del Poder Judicial como un acto imprescindible en la construcción de un Estado democrático de derecho». ${ }^{4}$

Partiendo de estas premisas, me pregunto cuál es el aporte que puede significar incorporar la categoría de género en la labor de los jueces y juezas a fin de avanzar de las solemnes declaraciones de derechos humanos a su acción efectiva y, en el caso de este artículo, desde el reconocimiento de la histórica y aún permanente situación de discriminación, opresión y subordinación en la que se encuentra la mitad de la humanidad: las mujeres, manteniendo respecto de ellas incumplidas las promesas de la Modernidad. ${ }^{5}$

En este punto es necesario aclarar el concepto de género. ${ }^{6}$ Para ello, me valdré de

1. El Estado constitucional, dirá Zagrebelsky (1995: 33), «está en contradicción con esta inercia mental».

2. Basado en la idea del «juez vinculado» a la que refiere Prieto (1993: 11-13), en cuanto debe subordinación a la ley, esto es, «de juez que ante todo viene obligado a fallar de acuerdo con ciertas normas o estándares preexistentes y cuya función es satisfacer unas expectativas que nacen, no de la confianza en sus cualidades personales, sino de unas prescripciones conocidas por las partes».

3. Atienza (2017: 195-197) en este sentido se refiere a la cultura de los derechos como una de las grandes transformaciones de las últimas dos décadas — junto con lo que denomina el «giro argumentativo»-, que hunde sus raíces en la Modernidad, lo que convierte los derechos humanos en un componente esencial del derecho (nuestros derechos) y de nuestra visión del mundo.

4. Suprema Corte de Justicia de la Nación, «Protocolo para juzgar con perspectiva de género: Haciendo realidad el derecho a la igualdad», Cámara de Diputados de México, disponible en https://bit. $\mathrm{ly} / 2 \mathrm{rm}_{3} \mathrm{ftg}$.

5. En su libro Cuando el género suena cambios trae (una metodología para el análisis de género del fenómeno legal) —nombre del cual, en parte, me apropio en este artículo- Facio (1992: 39-47) reconoce que la discriminación, opresión y subordinación de las mujeres de todos los grupos o clases discriminadas «es una de las formas más antiguas de opresión de unos seres hacia otros». La posición subordinada que ocupa la mujer se debe a que la sociedad está basada en una estructura de género «que mantiene a las mujeres de cualquier sector o clase, subordinadas a los hombres/varones de su mismo sector o clase y, relativamente, con menos poder que todos los hombres/varones», lo que legitima la subordinación del sexo femenino en contraposición a la situación de privilegio del hombre/varón.

6. Categoría incorporada por el feminismo académico de los años setenta «como fruto del análisis constructivista de la teoría feminista, que entiende que lo femenino y lo masculino responden a cons- 
la caracterización de Facio y Fries (1999: 34-44), quienes sostienen que alude tanto a características y comportamientos, como a roles, funciones y valoraciones impuestas de manera dicotómica a cada sexo a través de los procesos de socialización, mantenidos y reforzados por la ideología e instituciones patriarcales, ${ }^{7}$ y que es un problema de discriminación contra las mujeres desde dos puntos de vista: por un lado, porque las características y roles asignados a las mujeres gozan de menor o ningún valor y, por el otro, porque los que la sociedad atribuye a los hombres son los mismas que le asignan al género humano, por lo que lo asume como representante de la humanidad toda, e invisibiliza la parcialidad de esa perspectiva que se erige como universal e imparcial, por lo que define a lo opuesto (las mujeres) como negación, como «lo otro». En seguida, reconocen que este concepto no es abstracto ni universal, en tanto se concreta en cada sociedad en contextos espaciales y temporales, y se redefine en forma constante a la luz de otras realidades como la clase, etnia y edad, entre otras.

El uso del género, como aquellas gafas de aumento, ${ }^{8}$ constituye una herramienta con potencial transformador en cuanto «excede del mero proceso de elaboración de las normas, atravesando de nuevo su aplicación e interpretación, y su planeamiento político, y su óptima implementación, neutraliza los estereotipos de género latentes en los mismos». ${ }^{9}$ En palabras de Casas y González (2012: 251), «el juez, a través de sus fallos, tiene la posibilidad de aminorar las asimetrías de poder entre las partes atendido el mérito del caso cuando, en concreto, aplica el derecho a los hechos».

Se vuelve una categoría que se rebela en contra del pacto patriarcal, al visibilizar las situaciones vivenciadas por la mitad de la población (las mujeres) y que, por otro, permite corregir los impactos diferenciados entre hombres y mujeres de las normas, de su interpretación y aplicación; reconoce y cuestiona los roles que se le han atribuido a las mujeres, que tienen un efecto flagrante asignándoles roles serviles, devaluando sus atributos y características, pudiendo ser condicionadas para internalizar

trucciones culturales que van más allá de la frontera puramente biológica entre los sexos» (Posada, 2007: 293).

7. Facio y Fries (1999: 45-46) otorgan una serie de características comunes en todos los sistemas patriarcales: i) es un sistema histórico y no es natural; ii) se fundamenta en el dominio del hombre ejercido a través de la violencia sexual contra la mujer que se instala, a través de la fuerza y el temor, en sus cuerpos; iii) el paradigma de lo humano, representado por el varón blanco, rico, en edad reproductiva, sin discapacidades físicas y heterosexual fija el punto más alto de la jerarquía respecto de cualquier otra condición o variable; y iv) las justificaciones que permiten las mantención del dominio patriarcal tienen su origen en las diferencias biológicas.

8. El «Cuaderno de buenas prácticas para incorporar la perspectiva de género en las sentencias» (2018) reconoce que la perspectiva de género «utilizada como unos lentes de aumento, permite poner el foco en las situaciones de discriminación, desigualdad y violencia que viven principalmente las mujeres» (página 60).

9. Juana María Gil Ruiz, «La interpretación de las normas bajo una perspectiva de género», Poderjudicial.es, disponible en https://bit.ly/2MvP7Fj. 
estereotipos negativos sobre sí mismas y para cumplir el rol asignado apropiado para su estatus, y que hunde sus raíces en el androcentrismo y el sexismo (Cook y Cusack, 2010: 1); supone además «un paso más en la consecución de la igualdad» (Carmona, 2015: 27), pues plantea exigencias de igualdad ya no solo formal, sino que material como «exigencias que conecta con la reflexión sobre los derechos de quienes habían sido «excluidos» de la representación liberal del titular de derechos y que justifica tratamientos diferentes en la existencia de diferentes necesidades que modulan el modo en lo que los derechos formalmente concedidos pueden ejercerse» (Barranco, 2010: 74), reconociendo que la estructura de género «se articula con otros sistemas de distancia o de relaciones asimétricas» (Fries y Matus, 1999: 47) como la etnia, la raza o la nacionalidad, entre otros, al reconocer que el género es una "categoría que se entrelaza con otras formas en el que el poder se ejerce contra esas individuales y comunidades excluidas» (Maturana, 2019: 635), incorporando el uso del paradigma de la interseccionalidad, ${ }^{10}$ que permite "poder comprender que el contexto es fundamental, siendo necesario su análisis crítico para entender la situación de la persona. Y es que este contexto está conformado por muchos factores que se entrecruzan todos o varios de ellos a la vez» (Serra, 2017: 79).

Será precisamente en estos aportes en que centraré el análisis de algunas de las sentencias de la Corte Interamericana de Derechos Humanos y en un fallo de la Corte Suprema chilena.

\section{El género suena en la jurisprudencia de la Corte Interamericana de Derechos Humanos}

Antes de detenerme en el análisis de las sentencias de la Corte IDH, es importante mencionar que en general el sistema interamericano de derechos humanos estuvo por largos años ajeno a la temática de género. Sobre este punto, Medina (2003: 911912) plantea que la idea de recurrir a un órgano internacional en búsqueda de apoyo no cruzó la mente de las mujeres - que sin bien no se han encontrado totalmente inactivas, tomará tiempo ejerzan sus derechos, porque para ello deben conocerlos, para luego superar el sentimiento de que son para el resto, además de percibir que tiene algún sentido ejercitarlos-y posiblemente, en el caso de que se hubiesen presentado

10. El concepto fue introducido en el debate por parte del movimiento feminista negro, en especial por Crenshaw (2012: 89-92) en un artículo a fines de los años ochenta, «para señalar las distintas formas en las que la raza y el género interactúan, y cómo generan múltiples dimensiones que conforman las experiencias de las mujeres negras [...]. La interacción del racismo y del sexismo en las vidas de las mujeres negras afectan sus vidas de maneras que no se pueden entender del todo mirando por separado las dimensiones de raza o género", se refiere a las intersecciones de las estructuras de poder —en el caso que investigó: raza, género y clase- y que «constituyen los elementos estructurales primarios que conforman las experiencias de muchas mujeres negras y latinas en casas de acogida». 
violaciones de los derechos humanos de la mujer, habrían sido rechazados por que la composición de la Comisión (hombres) que pueden no haber sido conscientes de que las violaciones de los derechos de la mujer podrían ser violados en formar diferentes respecto de los hombres, motivo supuesto que las mujeres no cuestionaron."

Aclarado lo anterior, y centrándome en dos aportes de la incorporación del género en las sentencias de la Corte IDH, me detendré a analizar el primer caso que incluye claramente una perspectiva de género: el caso Penal Castro con Perú, ${ }^{12}$ y el último: el caso Mujeres víctimas de tortura sexual en Atenco con México ${ }^{13} \mathrm{e}$ identificar los aportes de su reconocimiento y visibilización.

\section{Afectaciones particulares respecto de las mujeres y las violaciones de los derechos}

En el caso Penal Castro con Perú, la Corte IDH reconoció al inicio del análisis de la determinación de la violación del artículo 5 de la Convención Americana de Derechos Humanos $(\mathrm{CADH})$ que «especificará los efectos particulares de los hechos con respecto a las internas en general y a las internas embarazadas» (párrafo 275), como de los aspectos específicos de violencia contra la mujer (párrafo 276). La Corte desarrolla su línea argumentativa reconociendo que durante el operativo Mudanza 1, los internos (hombres y mujeres) fueron víctimas de tortura psicológica (párrafo 288), para luego de manera específica reconocer que en el caso de las internas embarazadas «experimentaron un sufrimiento psicológico adicional, ya que además de haber visto lesionada su propia integridad física, padecieron sentimientos de angustia, desesperación y miedo por el peligro que corría la vida de sus hijos» (párrafo 292), encontrándose protegidas no solo por el derecho consagrado en el artículo 5 de la $\mathrm{CADH}$, sino también del artículo 7 de la Convención para Prevenir, Sancionar y Erradicar la Violencia contra la Mujer, que señala de manera expresa el deber de los Estados de velar por que las autoridades y agentes estatales se abstengan de cualquier acción o práctica de violencia contra la mujer (párrafo 292).

Luego, en cuanto a los hechos ocurridos después del 9 de mayo, la Corte reconoció que sobre las embarazadas los «agentes estatales no tuvieron ninguna consideración respecto a la condición específica de éstas» (párrafo 298), lo que agrava la violación del artículo 5 (párrafo 300). Por último, en cuanto a los hechos ocurridos en los centros de salud, la Corte reconoció que si bien internos e internas fueron desnudados

11. Sobre este tema, véase también Fernández (2017: 2-4).

12. Sentencia del caso Penal Miguel Castro Castro con Perú, serie C núm. 160, fondo, reparaciones y costas, 25 de noviembre de 2006.

13. Sentencia del caso Mujeres víctimas de tortura sexual en Atenco con México, serie C núm. 371, excepción preliminar, fondo, reparaciones y costas, 28 de noviembre de 2018. 
y obligados a permanecer sin ropa (párrafo 304), lo cual es un trato violatorio de su dignidad (párrafo 305), dicha desnudez, respecto de las mujeres, «tuvo características especialmente graves» (párrafo 306). Con posterioridad, estableció que las internas al no permitir que se asearan y, en algunos casos, que para utilizar los servicios sanitarios debían ir acompañadas de un guardia armado, quien no les permitía cerrar la puerta y las apuntaba con el arma mientras hacían sus necesidades fisiológicas, significó que fueran víctimas de violencia sexual al ser constantemente observadas por hombres (párrafo 306), en un contexto en que las mujeres «se hallaban sujetas al completo control del poder de agentes del Estado, absolutamente indefensas, y habían sido heridas precisamente por agentes estatales de seguridad» (párrafo 307), lo que les causó «un constante temor ante la posibilidad de que dicha violencia se extremara aún más por parte de los agentes de seguridad, todo lo cual les ocasionó grave sufrimiento psicológico y moral, que se añade al sufrimiento físico que ya estaban padeciendo a causa de sus heridas» (párrafo 308).

La Corte además precisó que una de las internas fue víctima de una inspección vaginal dactilar (párrafo 309), acto constitutivo de una violación sexual (párrafo 310), el que reconoce como un acto ejecutado por agentes del Estado «especialmente grave y reprobable, tomando en cuenta la vulnerabilidad de la víctima y el abuso de poder que despliega el agente» (párrafo 311) y que por sus efectos — que se reconoce además que «tiene consecuencias físicas, emocionales y psicológicas devastadoras» (párrafo 311) - constituye tortura (párrafo 312), al tratarse de una práctica que, en el caso de conflictos armados, se «considera y practica para humillar al adversario» (párrafo 313), utilizada como un acto simbólico para humillar a la parte contraria (párrafo 223) y como medio de castigo y represión, y que además tiene por objetivo «causar un efecto en la sociedad a través de esas violaciones y dar un mensaje o lección» (párrafo 224). ${ }^{14}$

\section{Estereotipos de roles asignados a las mujeres que impactan} en la situación de discriminación

Siguiendo su línea jurisprudencial, en el caso Mujeres víctimas de tortura sexual en Atenco con México, la Corte IDH reconoció que los estereotipos de género - definido por primera vez en la sentencia del caso González y otras ( Campo Algodonero»)

14. La Corte lo ha reconocido también en las sentencias del caso Masacres de El Mozote y lugares aledaños con El Salvador, serie C núm. 252, fondo, reparaciones y costas, 25 de octubre de 2012, párrafo 165; caso Espinoza Gonzáles con Perú, serie C núm. 289, excepciones preliminares, fondo, reparaciones y costas, 20 de noviembre de 2014, párr. 226; y en el caso Mujeres víctimas de tortura sexual en Atenco con México, serie C núm. 371, excepción preliminar, fondo, reparaciones y costas, 28 de noviembre de 2018, párrafo 200. 
con México- ${ }^{15}$ como «una preconcepción de atributos, conductas o características poseídas o papeles que son o deberían ser ejecutados por hombres y mujeres respectivamente», por lo que resulta posible relacionar la subordinación de la mujer a prácticas basadas en estereotipos de género, y por tanto su creación y uso es una de las causas y consecuencias de la violencia contra la mujer (párrafo 213), por lo cual deben los Estados adoptar «medidas activas y positivas para combatir actitudes estereotipadas y discriminatorias», para activamente luchar contra estos prejuicios y garantizar a las mujeres una igualdad real, no bastando una actitud pasiva o la simple sanción posterior por parte del Estado, en cuanto los prejuicios y patrones socioculturales se encuentra profundamente arraigados y la inacción del Estado los refuerza e institucionaliza, lo cual genera y reproduce violencia contra la mujer (párrafo 218), lo que viola la prohibición general de discriminación contenida en el artículo 1.1 de la Convención (párrafo 220).

Es interesante cómo la Corte IDH relaciona la discriminación, prohibida en la $\mathrm{CADH}$ con los estereotipos de género, y que implican una situación de violencia en contra de las mujeres. Por otro lado, en sentencias previas la Corte reconoció que «no está validando dichos estereotipos y tan solo los reconoce y visibiliza para precisar el impacto desproporcionado de la interferencia generada por la sentencia de la Sala Constitucional», y que los estereotipos de género son incompatibles con el derecho internacional de los derechos humanos. ${ }^{16}$

15. Caso González y otras («Campo Algodonero») con México, serie C núm. 205, excepción preliminar, fondo, reparaciones y costas, 16 de noviembre de 2009, párrafo 401. Estereotipos que se reflejaron en los comentarios efectuados por los funcionarios hacia los familiares de las víctimas (párrafo 208), asociando en el fallo «la subordinación de la mujer a prácticas basadas en estereotipos de género socialmente dominantes y socialmente persistentes, condiciones que se agravan cuando los estereotipos se reflejan, implícita o explícitamente, en políticas y prácticas, particularmente en el razonamiento y el lenguaje de las autoridades de policía judicial, como ocurrió en el presente caso. La creación y uso de estereotipos se convierte en una de las causas y consecuencias de la violencia de género en contra de la mujer» (párrafo 401). Además, la Corte reconoció que, tanto la inacción estatal al comienzo de la investigación, como los estereotipos de género, y sus consecuencias respecto de la impunidad, «envía el mensaje de que la violencia contra la mujer es tolerada, lo que favorece su perpetuación y la aceptación social del fenómeno, el sentimiento y la sensación de inseguridad en las mujeres, así como una persistente desconfianza de éstas en el sistema de administración de justicia» (párrafo 40o). Respecto del tema de estereotipos de género, entre otras, la Corte se ha pronunciado en los casos Atala Riffo y niñas con Chile, serie C núm. 239, fondo, reparaciones y costas, 24 de febrero de 2012., párrafos 131, 138 y 140; caso Véliz Franco y otros con Guatemala, serie C núm 277, excepciones preliminares, fondo, reparaciones y costas, 19 de mayo de 2014, párrafo 401; caso Fornerón e hija con Argentina, serie C núm. 242, fondo, reparaciones y costas, 27 de abril de 2012, párrafo 94; y caso I.V. con Bolivia, serie C núm. 329, excepciones preliminares, fondo, reparaciones y costas, 30 de noviembre de 2016, párrafo 187.

16. Caso Artavia Murillo y otros (Fecundación in Vitro) con Costa Rica, serie C núm. 257, excepciones preliminares, fondo, reparaciones y costas, 28 de noviembre de 2012. párrafo 302. 


\section{El género suena en la Corte Suprema}

La Corte Suprema, ${ }^{17}$ al revocar la sentencia de primera instancia que rechazó el recurso de amparo a favor de Lorenza Cayuhán Llebul, víctima de coerción ilegítima por parte de funcionarios de Gendarmería durante el proceso de su parto, ${ }^{18}$ incluyó entre sus argumentos la perspectiva de igualdad de género y el reconocimiento de que estamos en un caso paradigmático de discriminación múltiple.

\section{Igualdad de género}

La Corte Suprema dirá que Gendarmería incurrió en un acto de discriminación en contra de la amparada en su condición de mujer, al haber los funcionarios asimilado este complejo y único proceso al de cualquier intervención quirúrgica, y aplicado las normas contenidas en las instrucciones de buen servicio sobre servicios hospitalarios y salidas al exterior, mientras que deberían, desde una perspectiva de género, haber considerado el impacto negativo que una aplicación no diferenciada de las normas y reglamento penitenciarios podía ocasionar en la amparada.

Lo que viene a cuestionar la Corte Suprema a Gendarmería es la aplicación por parte de los agentes del Estado de la clásica interpretación de la formula «hay que tratar igual a lo igual y desigual a lo desigual», 19 que la limita a una práctica de decisión universalista, es decir, trata a todas las $\mathrm{X}$ iguales en la medida en que son iguales en una serie de propiedades, caso en el cual se prevén las mismas consecuencias jurídicas. ${ }^{20}$ En este caso resulta aplicable la concepción positiva del derecho a la

17. Es importante destacar que, desde 2015, la Corte Suprema ha iniciado un proceso de institucionalización de la política de género en el Poder Judicial, dentro del cual destacan el «Estudio diagnóstico de la perspectiva de Igualdad de Género en el Poder Judicial» (2016), «Estudio sobre la situación de la protección de la maternidad y la paternidad al interior de Poder Judicial» (2018), el «Cuaderno de buenas prácticas para incorporar la perspectiva de género en las sentencias del Poder Judicial chileno» (2018) y la política contra el acoso sexual laboral (2018). Para un mayor análisis de la política de género, véase Maturana (2019: 712-799).

18. Para conocer los hechos, consúltese: sentencia del caso Pía Campos Campos por Lorenza Cayuhán Llebul con Gendarmería de Chile, rol 92.795-16, 1 de diciembre de 2016, considerandos 5 y 6.

19. A la que se refiere, entre otros autores, Alexy (2012: 347-382).

20. En tal sentido se ha pronunciado la Corte IDH, que de manera progresiva ha ido interpretando el derecho consagrado en el artículo 1.1 y 24 de la Convención Americana respecto de la igualdad, ya que su jurisprudencia reconoce que el principio fundamental de igualdad y no discriminación ha ingresado en el dominio del ius cogens, por lo que los Estados están obligados a abstenerse de ejecutar acciones que de cualquier manera vayan dirigidas, directa o indirectamente, a crear situaciones de discriminación de jure o de facto, junto con sostener que un trato es considerado discriminatorio cuando no tiene una justificación objetiva y razonable, es decir, cuando no persigue un fin legítimo y no existe una relación razonable de proporcionalidad entre los medios utilizados y el fin perseguido. Lo anterior conlleva a 
igualdad y no discriminación «relacionada con la obligación de los Estados de crear condiciones de igualdad real frente a grupos que han sido históricamente excluidos o que se encuentran en mayor riesgo de ser discriminados», ${ }^{21} \mathrm{y}$, por tanto, la obligación de «combatir las prácticas de ese carácter y establecer normas y otras medidas que reconozcan y aseguren la efectiva igualdad ante la ley de las personas». ${ }^{22}$

En este caso, Gendarmería aplicó la regla general de las medidas de seguridad, asimilando el especial proceso de parto al de cualquier intervención quirúrgica al que podría ser sometido un interno o interna privada de libertad, lo que hizo primar no el cuidado que requería la amparada en esas condiciones, sino el deber de evitar una eventual evasión o fuga, sin incorporar la propiedad mujer al momento de su parto como relevante para ordenar un trato diferenciado, en este caso, la aplicación diferencia del instructivo de buen servicio, y cuyo resultado fue un acto de discriminación por los agentes del Estado.

\section{El paradigma de la interseccionalidad}

Es interesante además cómo la Corte Suprema reconoce además que estamos frente a un caso paradigmático de interseccionalidad en la discriminación - por su condición de mujer, privada de libertad, parturienta y perteneciente al pueblo mapuche${ }_{, 3}^{23}$ que define como la «confluencia de factores entrecruzados de discriminación que se potencian e impactan negativamente en la amparada, pues ésta recibió un trato injusto, denigrante y vejatorio, dada su condición de mujer, gestante, parturienta, privada de libertad y perteneciente a la etnia mapuche» (considerando 16).

La razón que aporta la Corte permite, entonces, contextualizar a Lorenza Cayuhán, superando con ello la fórmula desde un sujeto «neutro», reconociendo una serie

declarar que no toda distinción de trato comporta una vulneración del derecho a la igualdad. En tal sentido las sentencias, de la Corte IDH en los casos V.R.P., V.P.C. $y$ otros con Nicaragua, serie C núm. 350, excepciones preliminares, fondo, reparaciones y costas, 8 de marzo de 2018. párrafo 289; Flor Freire con Ecuador, serie C núm. 315, excepción preliminar, fondo, reparaciones y costas, 31 de agosto de 2016, párrafo 109; Duque con Colombia, serie C núm. 310, excepciones preliminares, fondo, reparaciones y costas, 26 de febrero de 2016, párrafos 91-106; Velásquez Paiz y otros con Guatemala, serie C núm. 307, excepciones preliminares, fondo, Reparaciones y Costas, 19 de noviembre de 2015, párrafo 173; Norín Catrimán y otros (dirigentes, miembros y activista del pueblo indígena mapuche) con Chile, serie C núm. 279, fondo, reparaciones y costas, 29 de mayo de 2014. párrafo 200; Yatama con Nicaragua, serie C núm. 127 , excepciones preliminares, fondo, reparaciones y costas, 23 de junio de 2005, párrafo 185; y opinión consultiva OC-4/184, párrafo 56.

21. Caso Furlán y familiares con Argentina, serie C núm. 246, párrafo 267.

22. Caso V.R.P., V.P.C. y otros con Nicaragua, párrafo 289.

23. Otra categoría que la Corte Suprema reconoce como discriminación es la pertenencia de Lorenza Cayuhán a la comunidad mapuche (considerando 15). Sobre la sentencia y el desarrollo de la discriminación múltiple o interseccionales, véase Jopia y Labbé (2018: 437-452). 
de condiciones que se interrelacionaron para dar como resultado el trato injusto y degradante que recibió por parte de los agentes del Estado.

\section{Conclusiones}

Este artículo me ha permitido reflexionar acerca de la incorporación de la categoría género (perspectiva de género) en el momento de que se ejerce el acto cúlmine del ejercicio de la jurisdicción, esto es, cuando se dicta sentencia y posibilita que avancemos un paso más en el camino del reconocimiento, lo que trae una vieja aspiración feminista de plena ciudadanía de las mujeres.

La perspectiva de género ha sido recogida en diversas sentencias por la Corte Interamericana de Derechos Humanos, reconociendo la afectación diferenciada a las mujeres respecto de violaciones como la violencia sexual. En el caso de la sentencia de Lorenza Cayuhán, la Corte Suprema hace dos interesantes ejercicios: por un lado, reconoce que el trato no diferenciado constituyó un acto de discriminación, y por otro, contextualiza a la amparada reconociendo con ello que el género se entrecruza con las condiciones de vulnerabilidad.

\section{Referencias}

Alexy, Robert (2012). Teoría de los derechos fundamentales. Traducción de Carlos Bernal. $2^{a}$ edición. Madrid: Centro de Estudios Políticos y Constitucionales.

Atienza, Manuel (2017). Filosofía del derecho y transformación social. Madrid: Trotta. BARranco, María (2010). «El enfoque feminista de los derechos fundamentales desde la perspectiva de género». En Cristina Monereo y José Monereo (editores), Género y derechos fundamentales (pp. 49-85). Granada: Comares.

CARmona, Encarnación (2015). «La perspectiva de género y los derechos humanos». En Encarnación Carmona (editora), La perspectiva de género en los sistemas europeo e interamericano de derechos humanos (pp. 25-42). Madrid: Centro de Estudios Políticos y Constitucionales.

Casas, Lidia y Juan González (2012). «Estereotipos de género en sentencias del Tribunal Constitucional». En Anuario de Derecho Público Universidad Diego Portales (2012): 250-272. Disponible en https://bit.ly/2ZvFLOV.

Соок, Rebecca, Simone Cusack (2010). Gender stereotyping. Transnational Legal Perspectives. Philadelphia: Pensilvania University Press.

CrenshaW, Kimberly (2012). «Cartografiando los márgenes: Interseccionalidad, políticas identitarias y violencia contra las mujeres de color». En Lucas Platero (editor), Intersecciones: cuerpos y sexualidades en la encrucijada (pp. 87-122). Barcelona: Bellaterra. 
FACio, Alda (1992). Cuando el género suena cambios trae: Una metodología para el análisis de género del fenómeno legal. 1. ${ }^{\mathrm{a}}$ ed. San José: Ilanud.

FACIO, Alda y Lorena Fries (1999). «Feminismo, género y patriarcado». En Alda Facio y Lorena Fries (editoras), Género y derecho (pp. 21-60). 1. ${ }^{a}$ edición. Santiago: Lom. FERNÁNDEZ, Mariano (2017). «Aproximación a las temáticas de género en la jurisprudencia interamericana». Revista Argentina de Teoría del Derecho 17: 1-22.

FrIEs, Lorena y Verónica Matus (1999). El derecho: Trama y conjetura patriarcal. 1. ${ }^{\mathrm{a}}$ ed. Santiago: Lom y La Morada.

JopIA, Valeria y Natalia Labbé (2018). «Discriminaciones múltiples y la recepción en el derecho interno: El caso de Lorenza Cayuhán, comentario a la sentencia rol n. ${ }^{\circ}$ 92795-2019 de la Corte Suprema». Estudios Constitucionales 16 (1): 437-452. DOI: 10.4067/So718-52002018000100437.

Maturana, Pilar (2019). «Los derechos de las mujeres en cien años de cuentas públicas, un camino que recién comienza». En 100 años de cuentas públicas (pp. 6328oo). Santiago: Corte Suprema.

Medina, Cecilia (2003). «Derechos humanos de la mujer: ¿Dónde estamos ahora en las Américas?». En A. Manganas (editor), Essays in honour of Alice YotopoulosMarangopoulos (pp. 907-930). Volumen 2. Traducción del Centro de Derechos Humanos. Santiago: Facultad de Derecho Universidad de Chile.

PosadA, Luisa (2007). «El pensamiento de la diferencia sexual: el feminismo italiano. Luisa Muraro y el orden simbólico de la madre». En Celia Amorós y Ana de Miguel (coordinadoras), Teoría feminista: De la Ilustración a la globalización, del feminismo liberal a la posmodernidad (pp. 289-317). 2. a edición. Madrid: Minerva.

Prieto, Luis (1993). Ideología e interpretación jurídica. Madrid: Tecnos.

Suprema Corte de Justicia de la Nación (2013). Protocolo para juzgar con perspectiva de género: Haciendo realidad el derecho a la igualdad (2013). 1. ${ }^{a}$ edición. Ciudad de México: Suprema Corte de Justicia de la Nación.

Serra, María (2017). Mujeres con discapacidad: Sobre la discriminación y opresión interseccional. Madrid: Dykinson.

Zagrebelsky, Gustavo (1995). El derecho dúctil: Ley, derechos y justicia. Traducción Marina Gascón. Madrid: Trotta.

\section{Sobre la autora}

Pilar Andrea Maturana Cabezas es abogada. Licenciada en Ciencias Jurídicas de la Universidad Católica del Norte, Chile. Jueza del Tribunal de Familia de Colina. Directora de la Asociación de Magistradas Chilenas. Máster en Derechos Fundamentales de la Universidad Carlos III de Madrid, España. Máster en Argumentación Jurídica de la Universidad de Alicante, España. Experta universitaria en pueblos in- 
dígenas, derechos humanos y cooperación internacional de la Universidad Carlos III de Madrid, España. Diploma en Recurso de Protección: Dogmática y Jurisprudencia, de la Facultad de Derecho de la Universidad de Chile. Diploma en Argumentación Jurídica y Razonamiento Probatorio, Instituto de Estudios Judiciales. Integrante de la Comisión de Derechos Humanos y Género de la Asociación Nacional de Magistrados. Visitante profesional de la Corte Interamericana de Derechos Humanos. Su correo electrónico es pilarcabezas@gmail.com. 